\title{
Search for a Low Mass Standard Model Higgs Boson with the ATLAS Detector at the LHC
}

\author{
Bertrand Laforge ${ }^{\mathrm{a} b}$ \\ ${ }^{a}$ Laboratoire de Physique Nucléaire et de Hautes Energies, UMR 7585, CNRS/IN2P3, \\ Université Pierre et Marie Curie (UPMC) et Université Denis Diderot (UDD), Paris, France \\ ${ }^{b}$ On behalf of the ATLAS collaboration
}

\begin{abstract}
In 2011, the ATLAS experiment collected $4.9 \mathrm{fb}^{-1}$ of proton-proton collision data at $\sqrt{\mathrm{s}}_{\mathrm{s}}=7 \mathrm{TeV}$ provided by the Large Hadron Collider. This data set allowed the search for a Standard Model Higgs Boson up to $1 \mathrm{TeV}$. In this paper, the focus is put on the searches of a low mass Higgs in the range (110-150) GeV. Using the 2011 ATLAS dataset, the combination of all the channels showed that a SM Higgs boson was restricted to live in the region (116.6119.4) GeV and (122.1-129.2) GeV. Importantly, an excess of events has been observed around $126 \mathrm{GeV}$ with a statistical significance of 2.9 standard deviations. The present document describes the final results published by ATLAS based on the 2011 dataset alone.
\end{abstract}

Keywords: ATLAS, Higgs boson, LHC, Low Mass.

PACS: $14.80 . \mathrm{Bn}$

\section{INTRODUCTION}

The Standard Model (SM) of particle physics is the model of elementary particles and fields that describes successfully the results in particle physics produced by several experiments since more than 50 years. Nevertheless, one of the key ingredients of this model, the Higgs mechanism introduced to explain the Electroweak Symmetry breaking $[2,3,4,5,6]$, has not been verified experimentally. The main prediction of this mechanism is the existence of a new spin 0 boson called the SM Higgs boson.

After intensive but inconclusive searches of this new boson at LEP $[8,9]$ and TeVatron [10], the Large Hadron Collider (LHC) at CERN (Geneva) is now the machine at which this research is ongoing $[10,11]$. In the present paper the search of a low mass SM Higgs boson using the ATLAS detector is reported based on the full 2011 dataset [1].

\section{LHC OPERATIONS AND ATLAS DATA TAKING IN 2011-2012}

The ATLAS detector is a multipurpose experiment $[12,13,14]$ installed on the LHC [15] at CERN, Geneva. LHC provides high intensity proton beams that collide at the center of the detector. In 2011, ATLAS collected a dataset of $4.9 \mathrm{fb}^{-1}$ (after quality selection) at $\sqrt{s}_{\mathrm{s}}=7 \mathrm{TeV}$. 


\section{LOW MASS HIGGS SEARCH AT ATLAS IN 2011}

At the LHC, SM Higgs boson is produced through several production mechanisms (gluon-gluon fusion, vector boson fusion and associated production) and it decays to a large number of final states whose branching ratios depend on the hypothesized Higgs boson mass. The channels sensitive to a low mass SM Higgs boson $\left(110 \mathrm{GeV}<\mathrm{m}_{\mathrm{H}}<\right.$ $150 \mathrm{GeV}$ ), because of a favorable production cross-section times branching ratio $(\sigma x B R)$ and/or a high signal over background ratio $(\mathrm{S} / \mathrm{B})$, are given in Table 1 . In this paper is presented a combination of the results of the search of a SM Higgs in several channels, using up to $4.9 \mathrm{fb}^{-1}$ of data taken in 2011. Each channel is analyzed using categories based either on event physical properties or on detector performance considerations (number of jets, localization of leptons/photons in the detector...) to better optimize the analysis sensitivity [1].

TABLE 1. List of channels used in the ATLAS combined Higgs search at low mass. The dataset used to analyze each individual channel and the reference paper where each individual analysis is described in details are given. $\mathrm{V}$ is used for $\mathrm{Z}$

or $\mathrm{W}$ bosons. The symbol 1 is used for e or $\mu$ leptons while the symbol $v$ represents a neutrino. $\tau_{\text {lep, }}, \tau_{\text {had }}$ refer to the leptonic or hadronic decay of the $\tau$ lepton.

\begin{tabular}{|c|c|c|c|c|}
\hline Higgs Boson Decay & Subsequent Decay & $\begin{array}{c}\text { Higgs Mass sensitivity range } \\
{[\mathrm{GeV}]} \\
\end{array}$ & $\begin{array}{l}\int \mathcal{L} \mathbf{d t} \\
{\left[\mathrm{fb}^{-1}\right]}\end{array}$ & Reference \\
\hline \multicolumn{5}{|c|}{$2011 \sqrt{\mathrm{s}}=7 \mathrm{TeV}$} \\
\hline$\overline{\mathrm{H}} \rightarrow \mathrm{ZZ}^{(*)}$ & 41 & $110-600$ & 4.8 & [16] \\
\hline \multirow{2}{*}{$\begin{array}{c}\mathrm{H} \rightarrow \gamma \gamma \\
\mathrm{H} \rightarrow \mathrm{WW}^{(*)}\end{array}$} & - & $110-150$ & 4.9 & [17] \\
\hline & lvlv & $110-300$ & 4.7 & [18] \\
\hline \multirow{3}{*}{$\mathrm{H} \rightarrow \tau \tau$} & $\tau_{\text {lep }} \tau_{\text {lep }}$ & \multirow{3}{*}{$110-150$} & \multirow{3}{*}{4.7} & \multirow{3}{*}{ [19] } \\
\hline & $\tau_{\text {lep }} \tau_{\text {had }}$ & & & \\
\hline & $\tau_{\text {had }} \tau_{\text {had }}$ & & & \\
\hline \multirow{3}{*}{$\mathrm{VH} \rightarrow \mathrm{Vbb}$} & $Z \rightarrow v v$ & \multirow{3}{*}{$110-130$} & 4.6 & \multirow{3}{*}{ [20] } \\
\hline & $\mathrm{W} \rightarrow \mathrm{lv}$ & & 4.7 & \\
\hline & $\mathrm{Z} \rightarrow 11$ & & 4.7 & \\
\hline
\end{tabular}

Among all those channels, two can provide an accurate mass measurement of a potential Higgs boson candidate, namely the diphoton and four leptons final states, thanks to the precise measurement of the full final state provided by the Inner Detector, the Electromagnetic Calorimeter and the external Muon Spectrometer.

The 4-lepton channel has a large $\mathrm{S} / \mathrm{B}$ but a small event rate whereas the diphoton final state has a $\mathrm{S} / \mathrm{B}$ of few percent and a sizable statistics at the integrated luminosity delivered by the LHC up to now. The $\mathrm{WW}^{(*)}$ channel is characterized by a large event rate but provides very little information about the mass itself because of the presence of undetected neutrinos in the final state that degrades the $\mathrm{WW}^{(*)}$ system mass reconstruction. Other channels are included but are less informative with the analyzed data sample since they have neither a large oxBR nor a very good $\mathrm{S} / \mathrm{B}$. In these analyses, backgrounds are measured from data whenever possible or Monte Carlo (MC) based estimated using up-to-date theoretical predictions. The accuracy of MC predictions is usually validated in signal free control regions to access systematics. A 
huge effort has been put to estimate all the possible systematic effects that could affect the measurement as described in details in [1] including background modeling.

Figure 1 presents the key distributions of the two precision channels, namely the diphoton invariant mass (1-a) and the 4-lepton invariant mass (1-b), and exhibits a modest excess of events around $m_{H}=125 \mathrm{GeV}$.

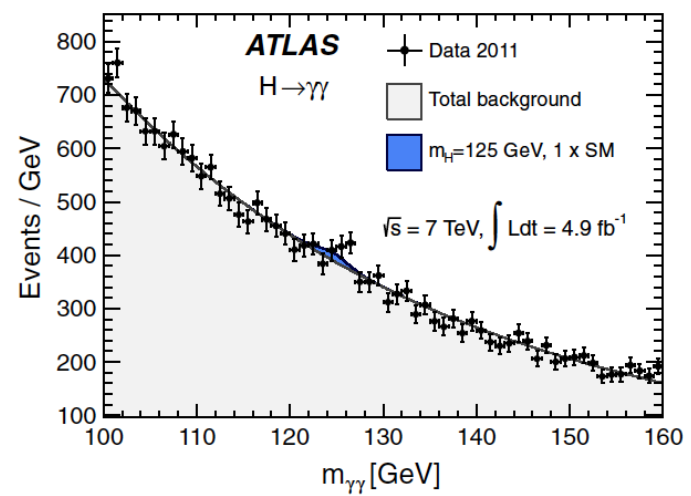

(a)

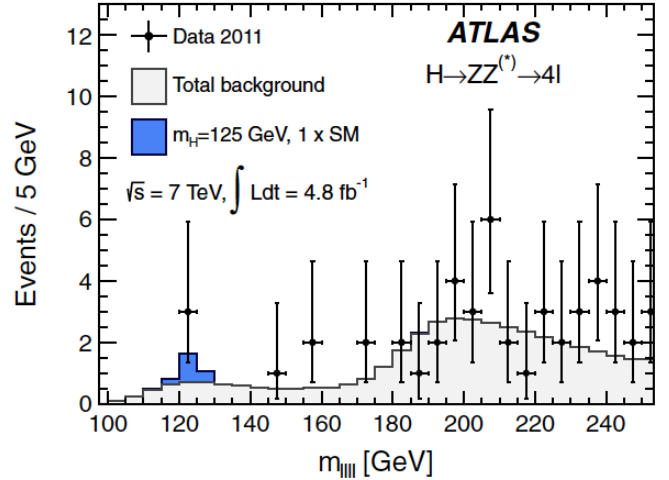

(b)

FIGURE 1. (a) Diphoton invariant mass and (b) 4-lepton invariant mass measured by ATLAS. The precise description of the selection of the various final states and of the background estimates can be found with great details in $[16,17]$.

The statistical significance of this excess is estimated using a maximum likelihood fit method [1] where the magnitude of the signal yield $(\mu)$ in units of SM cross-section is computed for each possible mass.

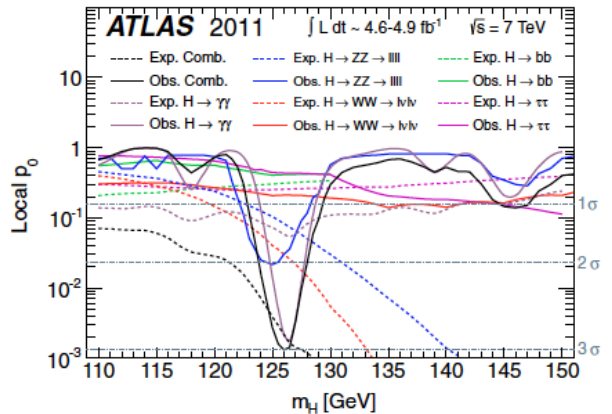

(a)

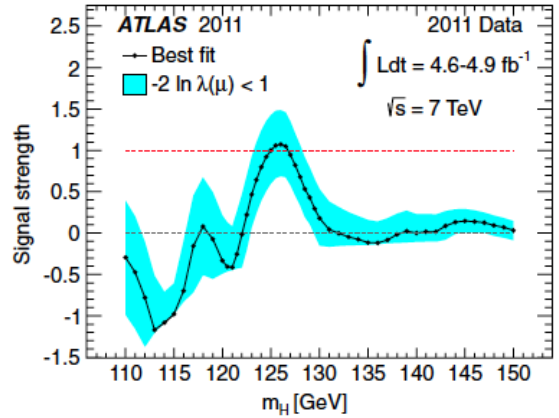

(b)

FIGURE 2. (a) Evolution of the probability that the SM background fluctuates more than the data as a function of the hypothesized SM Higgs mass $m_{H}[1]$ (b) Values of the signal magnitude in units of SM Higgs production cross-section as a function of $\mathrm{m}_{\mathrm{H}}$ [1].

Figure 2-a shows the probability that SM background fluctuates at least as much as the data. This probability shows a clear minimum at $\mathrm{m}_{\mathrm{H}}=126 \mathrm{GeV}$ corresponding to a statistical fluctuation of 2.9 standard deviations (local probability) [1]. Figure 2-b shows the values of $\mu$ as a function of the Higgs boson mass. The value $\mu=1.1 \pm 0.4$ found at $m_{H}=126 \mathrm{GeV}$ is compatible with the SM Higgs model prediction $(\mu=1)$ but 
more data are needed to understand if we are close to a discovery or only if we are facing a large statistical fluctuation.

The full ATLAS 2011 data sample allows also to set exclusion limits to the existence of a SM model Higgs in a wide mass range: 95\% confidence level (CL) exclusion regions extend from (111.4-116.6) GeV, (119.4-122.1) GeV, and (129.2-541) GeV. The mass region (130.7-506.0) GeV is excluded at the 99\% CL. Figure 3 -a shows the expected limits over the full mass range whereas Figure 3-b shows the observed limits in the low mass region.
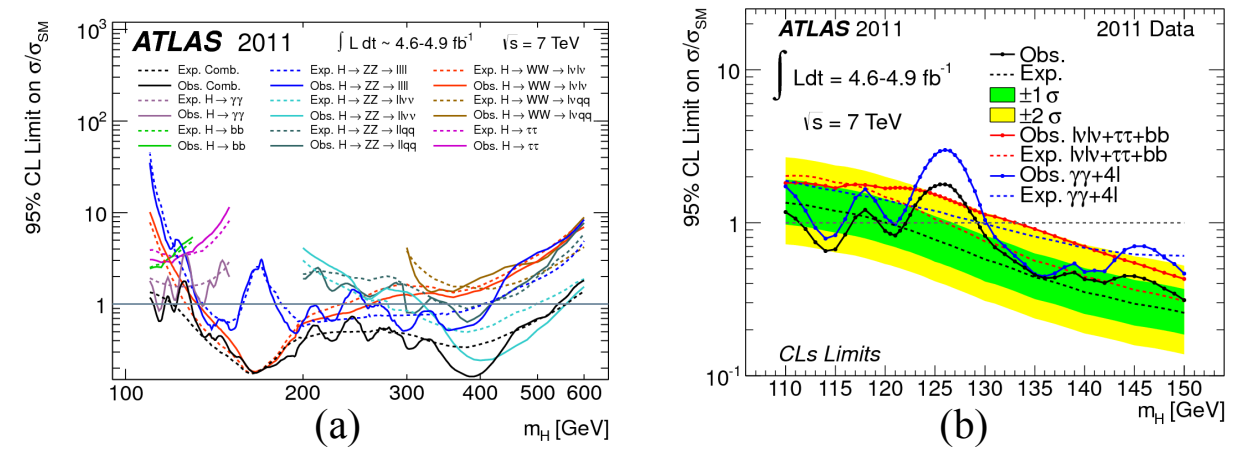

FIGURE 3. (a) Expected $95 \%$ CL limits of individual channels and their combination as a function of a possible SM Higgs mass for ATLAS 2011 dataset [1] (b) Details of the observed and expected CL limits as a function of $\mathrm{m}_{\mathrm{H}}$ in the low mass region for the combination for the different channels with the 2011 dataset [1].

At the time of writing of this document, the results on the SM Higgs boson search have been updated using up to $5.8 \mathrm{fb}^{-1}$ of proton-proton collision data taken in 2012 at $\sqrt{\mathrm{s}}_{\mathrm{s}}=8 \mathrm{TeV}$. Combining the results obtained with the new data with those from 2011, an excess of events around $\mathrm{m}_{\mathrm{H}}=126 \mathrm{GeV}$ is observed with a significance of 5.9 standard deviations. A detailed report can be found here [21].

More data are needed to measure with accuracy the physics properties of this new particle, to understand if this is indeed the Higgs boson predicted by the Standard Model.

\section{REFERENCES}

1. ATLAS collaboration, Combined search for the Standard Model Higgs boson in pp collisions at $\sqrt{s}_{s}=$ 7 TeV with the ATLAS detector, Physical Review D 86, 032003 (2012).

2. F. Englert and R. Brout, Broken symmetry and the mass of gauge vector mesons, Phys. Rev. Lett. 13 (1964) 321.

3. P. W. Higgs, Broken symmetries, massless particles and gauge fields, Phys. Lett. 12 (1964) 132.

4. P. W. Higgs, Broken symmetries and the masses of gauge bosons, Phys. Rev. Lett. 13 (1964) 508.

5. G. S. Guralnik, C. R. Hagen, and T. W. B. Kibble, Global conservation laws and massless particles, Phys. Rev. Lett. 13 (1964) 585.

6. P. W. Higgs, Spontaneous symmetry breakdown without massless bosons, Phys. Rev. 145 (1966) 1156. 
7. ALEPH, CDF, DØ, DELPHI, L3, OPAL, SLD Collaborations, the LEP Electroweak Working Group, the Tevatron Electroweak Working Group, and the SLD electroweak and heavy flavour groups, Precision Electroweak Measurements and Constraints on the Standard Model, CERN-PHEP-2010-095 (2010), arXiv:1012.2367 [hep-ex].

8. ALEPH, DELPHI, L3 and OPAL Collaborations, The LEP Working Group for Higgs boson searches, Search for the standard model Higgs boson at LEP, Phys. Lett. B 565 (2003) 61.

9. TEVNPH (Tevatron New Phenomena and Higgs Working Group), CDF and D0 Collaborations, Combined CDF and DO Upper Limits on Standard Model Higgs Boson Production with up to 8.6 $\mathrm{fb}-1$ of Data, arXiv:1107.5518 [hep-ex]. Presented at EPS 2011 Conference.

10. CMS Collaboration, Combined results of searches for the standard model Higgs boson in pp collisions at $s=7 \mathrm{TeV}$, arXiv:1202.1488; CMS-HIG-11-032, Phys. Lett. B 710 (2012) 26-48

11. ATLAS Collaboration, Combined search for the Standard Model Higgs boson using up to $4.9 \mathrm{fb}^{-1}$ of pp collision data at $\sqrt{s}_{s}=7$ TeV with the ATLAS detector at the LHC, arXiv:1202.1408 [hep-ex]. Phys. Lett. B 710 (2012) 49-66.

12. ATLAS Collaboration, ATLAS: letter of intent for a general-purpose pp experiment at the large hadron collider at CERN, CERN-LHCC-92-004 (1992).

13. ATLAS Collaboration, The ATLAS Collaboration, ATLAS Technical Proposal for a GeneralPurpose pp Experiment at the Large Hadron Collider at CERN, CERN-LHCC-94-43 (1994).

14. ATLAS Collaboration, The ATLAS Experiment at the CERN Large Hadron Collider, JINST 3 (2008) S08003.

15. L. Evans and P. Bryant (Eds.), LHC Machine, JINST 3 (2008) S08001.

16. ATLAS Collaboration, Search for the Standard Model Higgs boson in the decay channel $H \rightarrow Z Z^{(*)} \rightarrow 4 \ell$ with $4.8 \mathrm{fb}^{-1}$ of pp collisions at $\sqrt{s}_{s}=7 \mathrm{TeV}$ with ATLAS, Phys. Lett. B710 (2012) 383-402, arXiv:1202.1415 [hep-ex].

17. ATLAS Collaboration, Search for the Standard Model Higgs boson in the diphoton decay channel with $4.9 \mathrm{fb}^{-1}$ of ATLAS data at $\sqrt{s}_{\mathrm{s}}=7 \mathrm{TeV}$, Phys. Rev. Lett. 108 (2012) 111803, arXiv:1202.1414 [hep-ex].

18. ATLAS Collaboration, Search for the Standard Model Higgs boson in the $H \rightarrow W W^{(*)} \rightarrow l v l v$ decay mode with $4.7 \mathrm{fb}^{-1}$ of ATLAS data at $\sqrt{s}_{\mathrm{s}}=7 \mathrm{TeV}$, ATLAS-CONF-2012-060 (2012). https://cdsweb.cern.ch/record/1454675

19. ATLAS Collaboration, Search for the Standard Model Higgs boson in the $H \rightarrow \tau \tau$ decay mode in $\sqrt{s}_{s}$ = 7 TeV pp collisions with ATLAS, arXiv:1206.5971v1 [hep-ex]

20. ATLAS Collaboration, Search for the Standard Model Higgs boson produced in association with a vector boson and decaying to a b-quark pair with the ATLAS detector at the LHC, arXiv:1207.0210v1 [hep-ex]

21. ATLAS collaboration, Observation of a new particle in the search for the Standard Model Higgs boson with the ATLAS detector at the LHC, 2012, arXiv:1207.7214, accepted by Physics Letters B, (2012). 